\title{
Revealing yeast spore movement in confined space
}

\author{
J.L.F. Kock ${ }^{a}{ }^{*}$, C.J.Strauss ${ }^{a}$, E.E. Pretorius ${ }^{a}$, C.H.Pohl ${ }^{a}$, \\ A.S. Bareetseng ${ }^{\text {a }}$, P.J. Botes ${ }^{a}$, P.W.J.van Wyk ${ }^{b}$, S.W. Schoombie \\ and S. Nigam ${ }^{\text {d }}$
}

S OME YEASTS PRODUCE SEXUAL SPORES (ascospores) in a variety of shapes and surface ornamentations. These intriguing structures have hitherto been used only in yeast classification. Here, we propose the likely primary function of spore shape and ornamentations, in water-driven movement, as aiding the dispersal of the spores from enclosed containers (asci). This interpretation of the mechanics involved might find application in nano-, aero- and hydro-technologies with the re-scaling of these structures.

\section{Introduction}

Through sexual reproduction, ${ }^{1}$ some yeasts produce microscopic containers (asci) that enclose mainly water and spores (ascospores) of many different shapes and various nano-scale surface ornamentations. ${ }^{2}$ Some spores are spherical with an equatorial ledge (like the planet Saturn), or resemble hats with a bole and brim, while others look like corkscrews, walnuts, spindles with whip-like appendages, needles, and hairy or warty balls. Until now, these structures were used to classify yeasts and little thought was apparently given to their possible function. ${ }^{2}$ Here, we outline the role of spore shape and lubricated, nano-scale surface ornamentations in water-driven spore release.

\section{The clue}

The first clue regarding an explanation of the function of spore morphology came from a discovery we reported in 1991. Using radiolabelled thin-layer chromatography, $\left[{ }^{1} \mathrm{H}\right] 2 \mathrm{D}-\mathrm{COSY} \mathrm{NMR}$, gas chromatography-mass spectrometry as well as infrared spectroscopy, we revealed that the polyunsaturated 3-hydroxy oxylipin, 3R-hydroxy-5Z,8Z,11Z,14Z-eicosatetraenoic acid (3R-HETE or 3-OH 20:4), was

aUNESCO-MIRCEN, Department of Microbial, Biochemical and Food Biotechnology, ${ }^{\mathrm{b}}$ Centre for Confocal \& Electron Microscopy, ${ }^{\circ}$ Department of Mathematics and Applied Mathematics, University of the Free State, P.O. Box 339, Bloemfontein 9300, South Africa.

${ }^{\mathrm{d} D e p a r t m e n t}$ of Gynecology and Obstetrics, Free University of Berlin, Germany.

*Author for correspondence.

E-mail: kockjl@sci.uovs.ac.za sensitive to acetylsalicylic acid (aspirin). ${ }^{3,4}$ This compound as well as other polyunsaturated 3-OH oxylipins, namely, 3-OH 14:2, 3-OH 14:3, 3-OH 20:3, 3-OH 20:4 and $3-\mathrm{OH} 20: 5$, are produced by the yeast Dipodascopsis uninucleata probably through incomplete $\beta$-oxidation. With the aid of polyclonal antibodies directed against 3-OH oxylipins (with different chain lengths and desaturation ${ }^{5}$ ), we observed that these oxylipins coat part of nano-scale (100-600 $\mathrm{nm}$ in diameter) ornamentations on the surfaces of many spore types. ${ }^{3}$ The fact that hydroxy oxylipins have excellent lubricating properties and are today used in high-quality motor oils and lubricants for, amongst others, jet engines, ${ }^{6}$ gave rise to the following question. Do these compounds have a similar lubricating function on the surfaces of spores? If so, why?

\section{Message in a bottle}

We found a message concerning this question when studying spore release from bottle-shaped containers (asci) in the yeast Dipodascopsis uninucleata. ${ }^{3,5,7}$ Here, spores with hooked surface ridges and linked in gear-like fashion within a bottle-shaped ascus, are covered in oily hydroxy oxylipins - resembling oil inside a gearbox. Strikingly, when a 3hydroxy oxylipin inhibitor, acetylsalicylic acid, was added during the cultivation of this yeast, it inhibited the production of the oxylipin and subsequent release of spores in a dose-dependent manner. ${ }^{4,7} \mathrm{We}$ concluded that spore release is oxylipin-dependent probably also through the latter's lubricating ability. This interpretation prompted us to investigate the mode of spore release further from bottle-shaped containers in the yeasts in the genus Dipodascus as well as in D. uninucleata.

Those who have attempted to remove marbles or beans from an open bottleshaped container will know that it is necessary, first, to invert and then shake the bottle in all directions (Fig. 1). In this way these objects are loosened, a prerequisite for sliding past each other under gravity for unhindered individual release through the narrow opening. When removing beans, it is necessary to shake the bottle even more vigorously, thereby also aligning them by chance near the narrow neck to pass, narrow end first, through the opening. Alignment prevents beans from turning sideways, which would block their release.

From the literature ${ }^{3,8}$ and microscopic observations, we found that D. uninucleata and Dipodascus have evolved sophisticated means that enable the dispersal of oxylipin-coated spherical and bean-shaped spores from asci without inverting or shaking. In this case, instead of gravitational or centripetal pull, spores are pushed by turgor pressure towards the narrow opening and then ejected (Fig. 2).

Studies of Dipodascopsis spores, ${ }^{3,5,7}$ which range from bean-like to ellipsoid in shape, suggest that oxylipin-coated, interlocked surface ridges and stretching across the length of the spore, are responsible for their alignment. Here, spores inside the container are positioned sideby-side in a column of linked clusters with elongated sides attached by interlocking hooked ridges in gear-like fashion and orientated mainly with one end towards the opening. We conclude that the hooked ridges form turbine-like structures at both ends (Fig. 3, see also Appendix), causing propeller-like rotation when the spores are pushed by water pressure towards the ascus opening. This rotational movement loosens the spores (by the unlocking of the hooked ridges) near

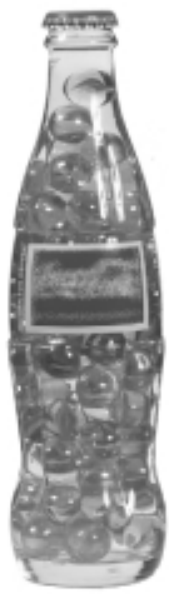

Fig. 1. Ever wondered how to remove marbles or beans through the narrow opening of a bottle without inverting or shaking it? This problem is solved by the yeast Dipodascopsis uninucleata var. uninucleata and by some species of Dipodascus, that produce bean-shaped, hooked spores (ascospores) ${ }^{2}$ and spherical sheathed spores, ${ }^{9}$ respectively. 

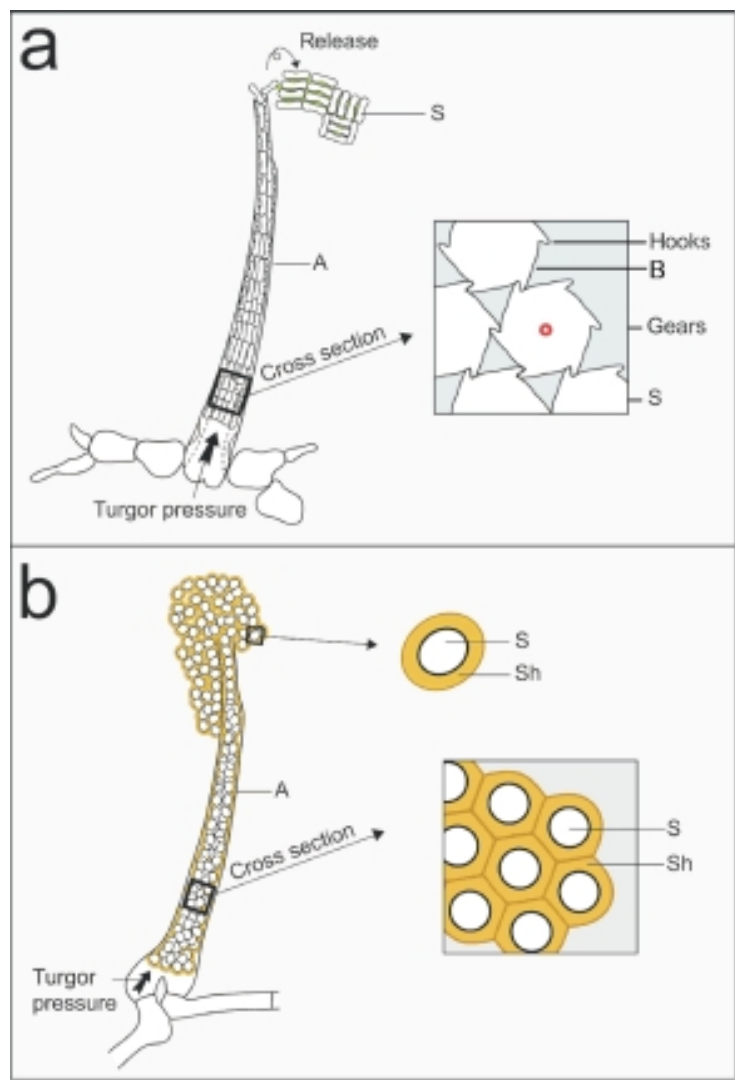

Fig. 2. Different spore release mechanisms in yeasts that ensure unhindered dispersal through narrow openings in bottle-shaped containers (asci). (a) Rotational spore release in Dipodascopsis uninucleata UOFS Y-2000 (Supplementary Movie 1). In this case, hooked, gear-like surface structures, stretching across the length of the spores, play an important role in loosening spores for unhindered forced release through the opening. Key: A, ascus; B, blade; S, spore. (b) Non-rotational spore release in Dipodascus sp. UOFS Y-1144. Here, flexible and compressible slimy sheaths enable spores to slip past each other when pressed towards the tapered tip without blocking the bottle-shaped container. Key: A, ascus; S, spore; Sh, sheath.

the container neck, which is necessary for their sliding past each other for eventual release. (Follow the effect of the rotation of one spore in gear-like fashion on the unlocking of attached neighbouring spores in the cross section shown in Fig. 2a; click here to view movie selection page.) Eventually, spores are released individually from the bottle-shaped ascus while rotating at about $1200 \mathrm{rpm}$ at approximately 110 length replacements per second (click here to view movie selection page). With some species of the genus Dipodascus, ${ }^{8,9}$ compressible, oxylipincoated sheathed surface structures and not gears are used to separate and loosen spherical spores in a similar bottle-shaped container before individual release under turgor pressure (Fig. 2b). These spores simply slide past each other when pressed towards the opening. We presume that more complex mechanics are needed to allow the effective release of these variously elongated spores compared to spherical sheathed spores, for

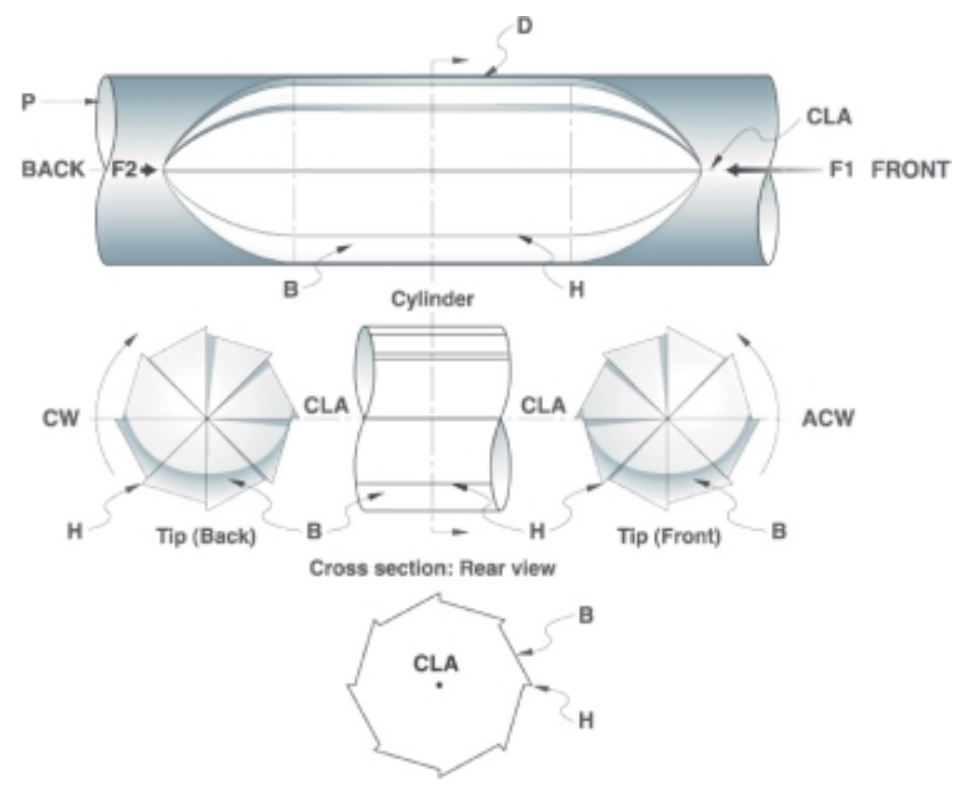

Fig. 3. A model, based on spore design and release in Dipodascopsis uninucleata, illustrating a device that we believe may keep (with minor changes) many kinds of pipelines clean while still in operation. Key: ACW, anticlockwise; B, blades; CLA, central longitudinal axis; D, device; F1, driving force; $\mathrm{F} 2$, backward force; $\mathrm{H}$, hooked ridges; $\mathrm{CW}$, clockwise; $\mathrm{P}$, pipe.

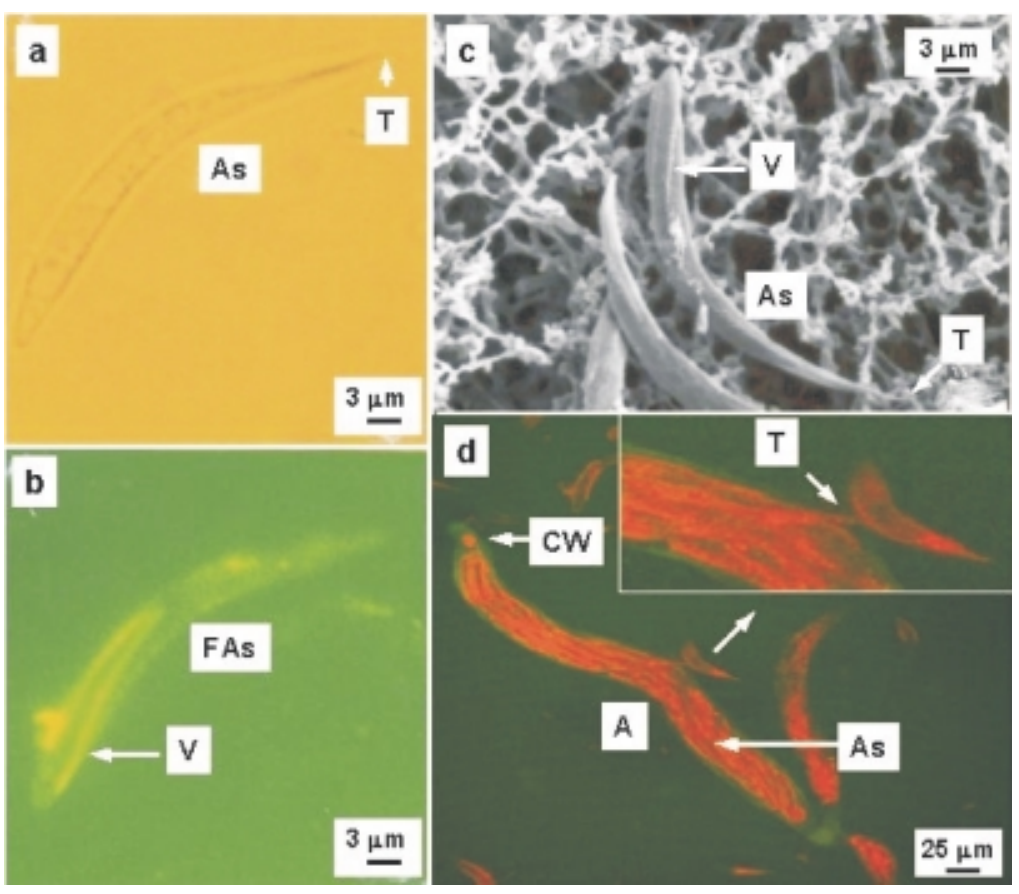

Fig. 4. Different images of sickle-shaped ascospores produced by Eremothecium ashbyi UOFS-Y 630 observed under various conditions: (a) light microscopy; (b) the subject in (a) viewed by immunofluorescence; (c) scanning electron micrograph; (d) confocal laser scanning micrographs indicating the spore's hydrophobic V-shaped fin structure (one leg of the $\mathrm{V}$ is approximately $600 \mathrm{~nm} \times 600 \mathrm{~nm} \times 20 \mu \mathrm{m}$, other leg is approximately $600 \mathrm{~nm} \times 600 \mathrm{~nm} \times 17 \mu \mathrm{m}$ ) and in the process of being released (spiked tip first). Key: A, ascus; As, ascospore; CW, cell wall (green), surrounding ascospores (red) stained with fluorescing Orange G; FAs, fluorescing ascospore stained with FITC anti-rabbit IgG; T, tip of spike; V, fluorescing V-shaped fins stained with FITC anti-rabbit IgG in (b) and V-fins observed in (c) using scanning electron microscopy. Amorphous remnants [in (b) and (c)] are attached to fins probably as a result of rupturing after passage through a narrow, self-created hole in cell wall.

which alignment and rotation are unnecessary.

\section{Yeast boomerangs}

We next asked ourselves what the functions of other spore shapes and oxylipin- lubricated surface ornamentations might be. Using gas chromatography-mass spectrometry according to the method described in ref. 4, we discovered a saturated 3-OH 14:0 compound (mass fragments:175 [ $\left.\mathrm{CH}_{3} \mathrm{O}(\mathrm{CO})-\mathrm{CH}_{2}-\mathrm{CHO}-\mathrm{TMSi}\right]$; 

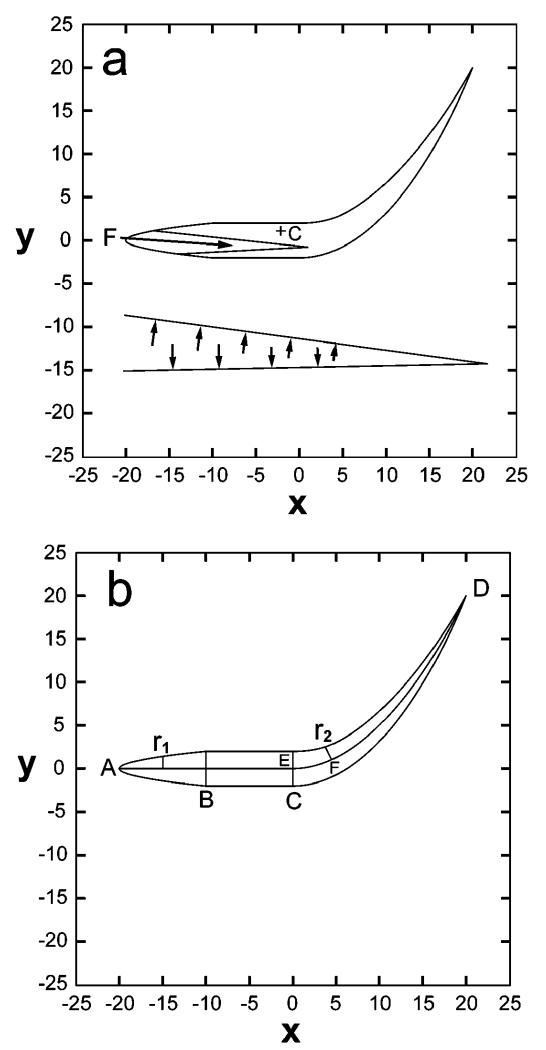

Fig. 5. A mathematical description, using elementary calculus, of a typical sickle-shaped spore of Eremothecium ashbyiiUOFS-Y 630. (a) Movement of spore by water pressure across fins; (b) determination of the centroid. The tapered front part of the spore was modelled by a solid of revolution formed by rotating a parabola about a horizontal axis. The middle part of the spore is represented by a cylinder, while the curved tail was modelled by first describing its curved centre line as an arc of a parabola, and then assuming the cross section perpendicular to the parabola to be a circle, the radius of which decreases in proportion to the arc length along the parabola. The position of the centroid of the simulated spore was then calculated, and found to be situated at $x=-2.14$ and $y=1.21$, as indicated in (a). We assume this centroid to coincide more or less with the centre of mass $\mathrm{C}(+)$ of the spore.

$\left.330\left[\mathrm{M}^{+}\right] ; 315\left[\mathrm{M}^{+}-15\right]\right)$ in the yeast Eremothecium ashbyii. In order to map the oxylipin's location in the yeast, we applied the same antibodies and immunofluorescence microscopy on cells in sexual mode as described in ref. 5 . The oxylipin was present as part of a V-shaped structure on sickle-shaped spores (Fig. 4a, b). With the aid of confocal laser scanning microscopy (Nikon TE 2000) to observe cells treated with antibody and fluorescine (FITC anti-rabbit IgG), ${ }^{5}$ we concluded that the hydrophobic V-shaped structure was present as a mirror image on both sides at the blunt end of an otherwise hydrophilic spore as indicated by differential ascospore staining. ${ }^{2}$ Scanning electron microscopy, according to the method used in ref. 10, showed this structure to be fin-like protuberances (Fig. 4c).

Next, we addressed the function of these fin-like structures and spore shape.

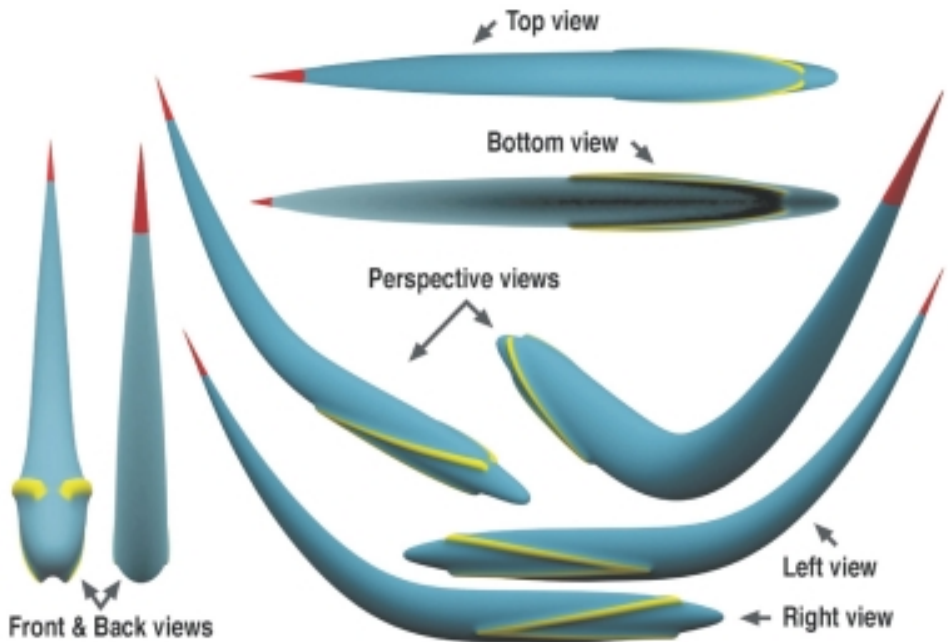

Fig. 6. A 3-D simulation, showing all sides, of the sickle-shaped spore of Eremothecium ashbyii UOFS-Y630.

Using microscopy, we discovered that spores are sometimes forced through the ascus with the spiked tip rupturing the ascus wall (Fig. 4d). Water pressure caused a boomerang movement when the blunt end was pushed forward with the spike leading the way in a circular motion. This happened only when micron-scale streams of water moved across the fins from the blunt end towards the tip of the spore. Mathematical modelling suggests a sharp build-up of pressure between fins, when water flows towards the blunt end and across the fins (from left to right in Fig. 5a, b). The pressure acts perpendicular to the fins (a, bottom). Because of their hydrophobic behaviour, there should be no viscous effects, that is, no forces acting parallel to the fins. Consequently, these pressures culminate in a resultant force across the spore, from left to right and slightly downwards, indicated by force vector $F$, thereby causing movement of the spore to the right. Since the line of force passes below the centre of mass at $\mathrm{C}(+)$, the spore will also tend to rotate anticlockwise, that is, in the direction of the spiked end, about $\mathrm{C}(+)$. In addition, there should be a tendency for water pressure to be greater at the left of the spore than on the right, since the spore gradually tapers towards the spike, that is, from the approximately $3-\mu \mathrm{m}$-diameter blunt end to the 2-nm-diameter spike. This shape should also enhance the boomerang effect. Furthermore, the shape of the fins (Fig. 5a) is such that they also act as hydrofoils when they start to move, creating a lifting force (as a result of a backward force on the slanted lower fins) on the spore, similar to the wings of an aircraft. Thus, the spore will start drifting to the right and slightly upwards (that is, closer to the cell wall), rotating anticlockwise until the spike reaches the ascus wall, where the latter may be ruptured and the spore pushed out of the cell by water pressure. In addition, fins lend stability to the blunt end, that is, they resist rotation when pushed by water flow, causing the spiked tip to reach the cell wall at the speed required for rupturing. Fins are also constructed in such a way that upon release through a selfpunctured narrow opening, the spearshaped end of the hydrophobic V structure exits first, thereby preventing spores becoming stuck to the cell wall. The relatively small height and width dimensions of the fins also support this argument, although the effective area offering water resistance is probably increased by their hydrophobic nature. We propose the formation of 'nanobubbles' through drying $^{11}$ at the fin-water interface, thereby increasing the relatively flat and thin fin surface area on the otherwise non-hydrophobic spore surface. This in turn increases the resistance of the fins to the water movement, which enhances overall spore stability and boomerang speed.

\section{Conclusions}

We believe that this report has only scratched the surface of water-driven spore movement in yeasts on a micrometre scale and that the mechanical implications of many spore shapes with a large number of different hydroxy oxylipin-lubricated, nano-scale surface ornamentations await similar explanation and elaboration.

Why did some yeasts evolve their characteristic spore movement with the beneficial consequence, so far as we can see, to escape from closed or partially closed containers? ${ }^{2}$ Of course, this would be important from a survival point of view, since without this ability, yeast 
spores would presumably not be able to disperse properly. The function of spore structure confined in persistent asci (that is, they seemingly do not release spores from these enclosures) ${ }^{2}$ should be investigated. We believe that if appropriate ultrastructural studies ${ }^{10}$ are conducted on yeasts with the aim of exposing spore surface ornamentations and not merely membrane structure, ${ }^{2}$ clues can be gained to reveal the mechanics behind the motion of nano-sized particles in fluids.

This experience with yeasts might now be profitably applied to other, non-related cells of different shapes and ornamentations such as blood components and plant seeds dispersed by wind and water, as well as in nano-, aero- and hydro-technologies (see Appendix).

This work is supported by the National Research Foundation, South Africa, and the Volkswagen Foundation, Germany (1/74643). We thank Mark Vermeulen of Serengeti Moon, and Christo Steyn and Kobus van Wyk for the illustrations.

1. Van der Walt J.P. (1999). Interpreting the automictic ascomycetous yeasts. S. Afr. J. Sci. 95, 440441.

2. Yarrow D. (1998). Methods for isolation, maintenance and identification of yeasts. In The Yeasts A taxonomic study, 4 th edn, eds C.P. Kurtzman and J.W. Fell, pp. 77-100. Elsevier, Amsterdam.

3. Kock J.L.F., Strauss C.J., Pohl C.H. and Nigam S. (2003). The distribution of 3-hydroxy oxylipins in fungi. Prostaglandins $\&$ Other Lipid Mediators 71, 85-96.

4. Van Dyk M.S., Kock J.L.F., Coetzee D.J., Augustyn O.P.H. and Nigam S. (1991). Isolation of a novel aspirin sensitive arachidonic acid metabolite 3 -hydroxy-5,8,11,14-eicosatetraenoic acid (3-HETE) from the yeast Dipodascopsis uninucleata UOFS-Y128. FEBS Lett. 283, 195-198.

5. Kock J.L.F., Venter P., Linke D., Schewe T. and Nigam S. (1998). Biological dynamics and distribution of 3-hydroxy fatty acids in the yeast Dipodascopsis uninucleata as investigated by immunofluorescence microscopy. FEBS Lett. 427, 345-348.

6. Johnson D.L. (1999). High performance 4-cycle lubricants from canola. In Perspectives on New Crops and New Uses, ed. J. Janick, pp. 247-250. ASHS Press, Alexandria, VA.

7. Kock J.L.F., Van Wyk P.W.J., Venter P., Coetzee D.J., Smith D.P., Viljoen B.C. and Nigam S. (1999). An acetylsalicylic acid sensitive aggregation phenomenon in Dipodascopsis uninucleata. Ant. $v$ Leeuwenhoek 75, 261-266.

8. Smith D.P., Kock J.L.F., Van Wyk P.W.J., Pohl C.H. Van Heerden E., Botes P.J. and Nigam S. (2003). Oxylipins and ascospore morphology in the ascomycetous yeast genus Dipodascus. Ant. v. Leeuwenhoek 83, 317-325.

9. De Hoog G.S., Smith M.Th. and Guého E. (1998) Dipodascus de Lagerheim. In The Yeasts - A Taxonomic study, 4th edn, eds C.P. Kurtzman and J.W. Fell, pp. 181-193. Elsevier, Amsterdam.

10. Van Wyk P.W.J. and Wingfield M.J. (1991) Ascospore ultrastructure and development in Ophiostoma cucullatum. Mycologia 83(6), 698-707.

11. Ball P. (2003). How to keep dry in water. Nature $423,25-26$.

\section{Appendix}

A basis for new technologies?

These observations of spore movements in confined spaces outlined above prompted us to scale-up spore structures and investigate some of their hydrodynamic properties, in constructed and to learn if similar movements could be replicated when magnified.

Based on the design of D. uninucleata spores, ${ }^{7}$ we devised a new generation of pipe-cleaning and drilling pigs (named after the screeching noise these metal devices make when forced through tight-fitting pipes). Here, we adapted spore shape and surface ornamentations to produce an instrument that we surmise can be used effectively (with minor changes to blade slant and orientation) to keep industrial pipelines clean (similar to pipe-cleaning pigs) while in operation, that is, by scraping and drilling movements (Fig. 3).

From mathematical modelling, we simulated movement of the ellipsoidal device (similar to spore shape) when pushed by fluid pressure through a close-fitting pipe (simulating ascus neck and forced spore movement) filled with moving liquid (simulating water movement through the ascus neck), while the latter caused enhanced rotation by turning both turbine-shaped ends of the device simultaneously in the same direction (simulating spore rotation), thereby exploiting the available liquid forces more effectively. Hooked ridges and blade orientation are shown on the device while moving, forced by liquid pressure, to the left through a close-fitting pipe (Fig. 3).

Rotation about a central longitudinal axis is the result of two forces. There are the driving force F1 and the backward acting force F2, caused when the device is pressed against the liquid present in the pipe. Both forces act simultaneously and in concert perpendicular to the slanted blades on both turbine-like tips in propeller fashion. These forces result in a net resultant movement of the device to the left, particular to see if useful devices could be

guided by the pipe, as well as an increase in anticlockwise rotation observed from the front if forces are correctly balanced. This is the reason for the high rotational release of spores. Rotation will have a scraping effect, thereby removing any deposit on the inside of the pipes, while the rotating tip at the back will perform a clockwise drilling movement. This device may prove useful when used routinely, while liquid is pumped through pipes, to prevent them from clogging up. A scaled-up model (30 000 times spore size) positioned in a closely fitted pipe, and subjected to water pressure from one side, confirmed the behaviour anticipated (Fig. 7).

Other scaled-up models (10000 times enlarged), simulating the sickle-shaped spore (Fig. 6) and subjected to water pressure from their blunt end, replicated the boomerang movement of spores observed within asci. The hydrophobic water-resistant properties of fins could not be tested, however, since these forces become significant only when exerted on small objects in restricted environments. ${ }^{11}$ Furthermore, many spores packed into a micrometre-sized ascus may well behave differently. We propose also that a similar water-driven spore movement occurs within vascular bundles of plants where this yeast acts as a pathogen (click here to view movie selection page).

This boomerang mechanism may in future inspire the design of environmentally driven miniature fleets of medical nanorobots as envisaged by some students of nanotechnology. Perhaps, this concept may prove useful in scouring clogged arteries through continuously blood-driven boomerang movements, thereby sweeping the inside of artery walls with spiked ends without damaging them.

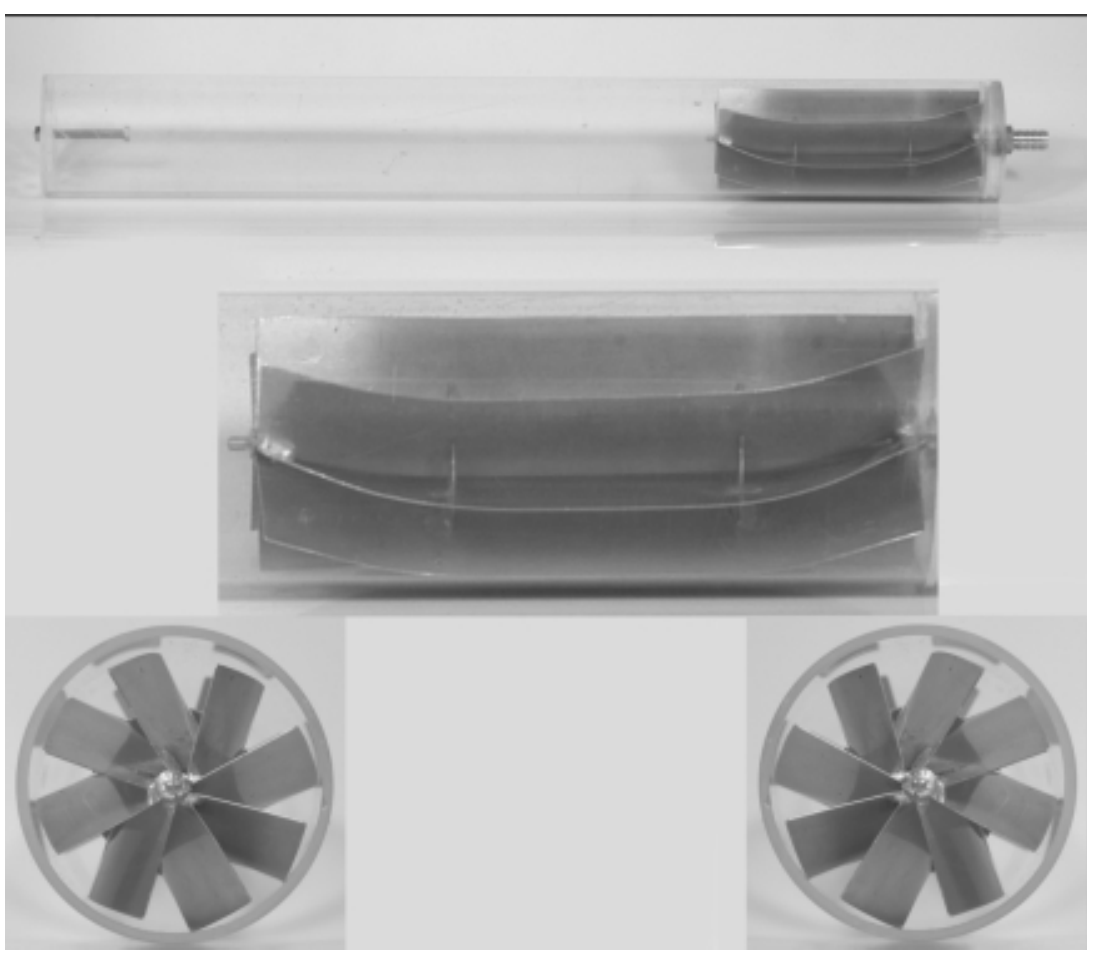

Fig. 7. An experimental pipe-cleaning device (measuring $75 \times 180 \mathrm{~mm}$ ) adapted from the design in Fig. 3 and constructed by the authors. The apparatus used for testing the device is shown at the top. Water pressure is applied to the pipe from the right-hand side. 

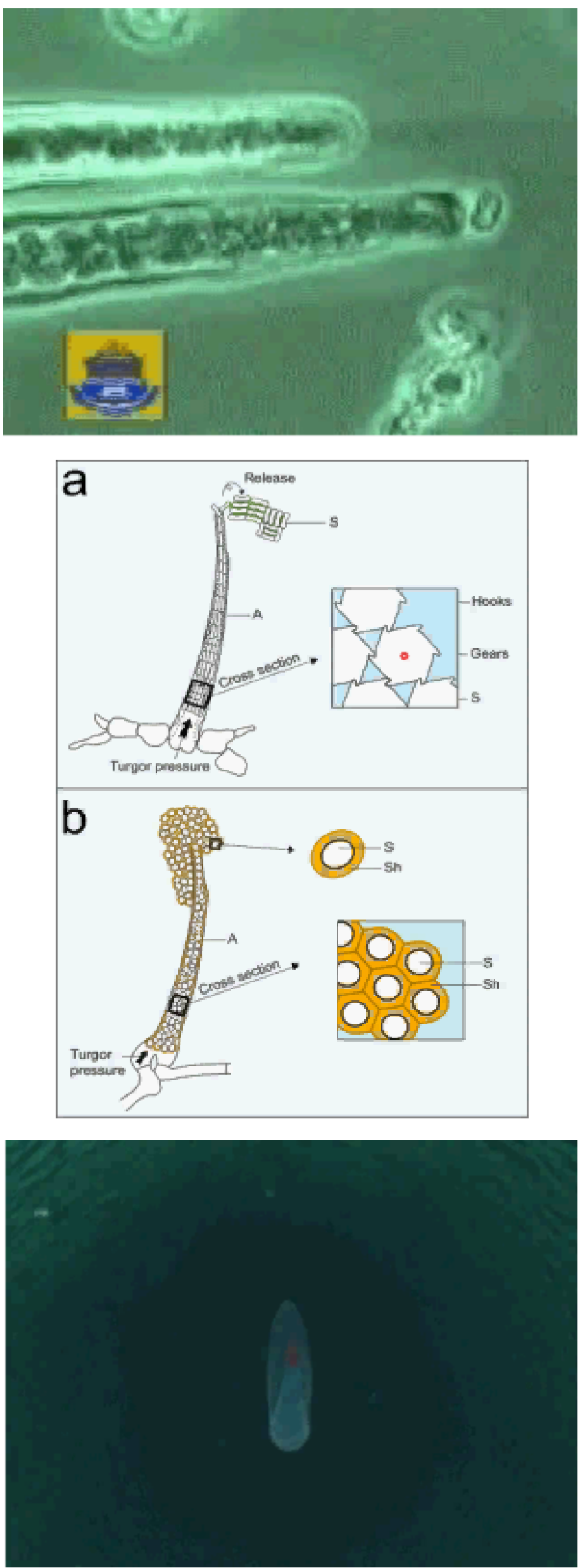

\section{Guide to movies}

Click on one of the images to view the associated movie. Apple's QuickTime Player 5 and Adobe ${ }^{\circledR}$ Acrobat 6 or later must be installed to view the movies. They can be downloaded from http://www.apple.com/quicktime/ and http://www.adobe.com/ acrobat/, respectively.

\section{Movie 1}

This movie is a video-enhanced microscopic presentation (slowed down 10 times) showing active cell (spore, approx. $1 \times$ $2.5 \mu \mathrm{m})$ release, one at a time while rotating, from the tip of a bottle-shaped container (ascus) (approx. $2 \mu \mathrm{m}$ in diameter) of Dipodascopsis UOFS Y-2000.

To return to text click here.

\section{Movie 2}

Different physical spore (ascospore) release mechanisms in yeasts ensuring unhindered dispersal through narrow openings of bottle-shaped containers (asci). a, Rotational sporerelease in Dipodascopsis uninucleata UOFS Y-2000. In this case, hooked, gear-like surface structures, stretching across the length of spores, play an important role in loosening spores for unhindered forced release through the opening. Key: A, ascus; $\mathrm{S}$, spore. $\mathbf{b}$, Non-rotational spore release in Dipodascus sp. UOFS Y-1144. Here, flexible and compressible lubricated sheaths enable spores to slip past each other when pressed towards the tapered tip without blocking the bottle-shaped container. Key: A, ascus; S, spore; Sh, sheath. See also Fig. 2.

\section{To return to text click here.}

\section{Movie 3}

This movie simulates movement of a sickle-shaped cell (ascospore) of Eremothecium ashbyii UOFS-Y 630 within an elongated container (ascus). Scene 1: Cell movement inside container with spiked tip (in red) moving towards screen. Hydrophobic V-shaped fins are in yellow. Scene 2: Cell moving along length of container with spiked tip leading the way. Cell direction same as water flow indicated by bubble movement. Scene 3: Side view of cell movement. Scene 4: Movement of spiked tip towards inside wall of container. Scene 5: Spiked tip piercing wall of container. Scene 6: Forced release of cell, spiked tip first through wall of container in boomerang style. Sometimes parts of container wall can remain attached to spiked tip, while the fins can tear as a result of moving through a tightfitting torn wall.

\section{To return to text click here.}

